\title{
Anomalías dentarias de unión: fusión dental
}

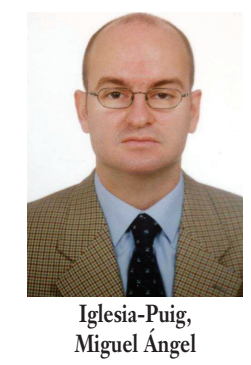

\author{
Union dental anomalies: Fused teeth
}

\section{Iglesia-Puig, Miguel Ángel* Arellano-Cabornero, Hlfonso** López-Areal García, Begoña***}

*Odontólogo. Ejercicio privado. Zaragoza

**Profesor de Patología y Terapéutica Dental. Universidad del País Vasco. ***Odontóloga. Ejercicio privado. Bilbao.

\section{Correspondencia}

Miguel Ángel Iglesia Puig

Clínica Bucodental MAIP

Residencial Paraíso 1, esc $\mathrm{B}, 1^{\circ} \mathrm{C}$ 50008 - Zaragoza

E-mail: driglesia@clinicamaip.net
Resumen: La fusión dental es una anomalía dentaria que consiste en la unión embriológica o en fases pre-eruptivas de dos o más gérmenes dentarios adyacentes por medio de dentina con el resultado de un diente único. Se presentan siete casos clínicos de fusiones dentarias, y se revisa el diagnóstico diferencial, las formas clínicas y el plan de tratamiento de esta anomalía.

Palabras clave: Anomalía dentaria de unión, Fusión dental.

Abstract: Fused teeth is a dental anomaly which consists in the union of at least two adjacent teeth by dentin, with the result of a unique teeth. Seven clinical cases of dental fusion are presented, and differential diagnosis, clinical patterns and treatment planning of fused teeth are reviewed.

Key words: Union dental anomaly, Fused teeth.

\begin{tabular}{ccc}
\hline $\begin{array}{c}\text { Fecha recepción } \\
12-6-2003\end{array}$ & Fecha última revisión & Fecha aceptación \\
\hline
\end{tabular}

BIBLID [1138-123X (2005)10:2; marzo-abril 125-240]

Iglesia-Puig A, Arellano-Cabornero A, López-Areal Carcía B. Anomalías dentarias de unión: fusión dental. RCOE 2005;10(2):209-214. 


\section{Introducción}

La fusión dental es una anomalía anatómica dentaria que consiste en la unión embriológica 0 en fases preeruptivas de dos o más gérmenes dentarios adyacentes por medio de dentina con el resultado de un diente único. En ocasiones pueden incluso compartir la cámara pulpar, aunque generalmente los dientes fusionados presentan dos cámaras pulpares ${ }^{1-3}$. Los dientes afectados erupcionan ya fusionados, efectuándose la fusión a lo largo de dientes situados en el mismo plano, pudiendo ser total, o limitada a la corona o a la raíz ${ }^{4}$.

Esta anomalía se da con mayor frecuencia en la dentición temporal que en la dentición permanente ${ }^{1,5,6}$. Según autores ocurre con más frecuencia en incisivos inferiores', aunque otros afirman que generalmente afectan a los incisivos superiores, sea como fusión del central y el lateral, sea como unión de un incisivo normal y un lateral supernumerario². En la literatura se establece una clara predilección de localización en las zonas anteriores de los maxilares, involucrando con mayor frecuencia los incisivos y los caninos en la fusión dentaria ${ }^{4,5,7-70}$, y afectándose raramente los premolares y molares $^{6}$. Se han descrito casos de fusiones bilaterales, siendo éstas también más frecuentes en dentición temporal y en el maxilar inferior?

La morfología en los dientes fusionados puede ser normal, con las variaciones derivadas del proceso de fusión. En la cara vestibular aparece un surco vertical, más o menos marcado, que indica la línea de fusión de ambos dientes; por palatino pueden presentarse dos cíngulos o uno sólo que se

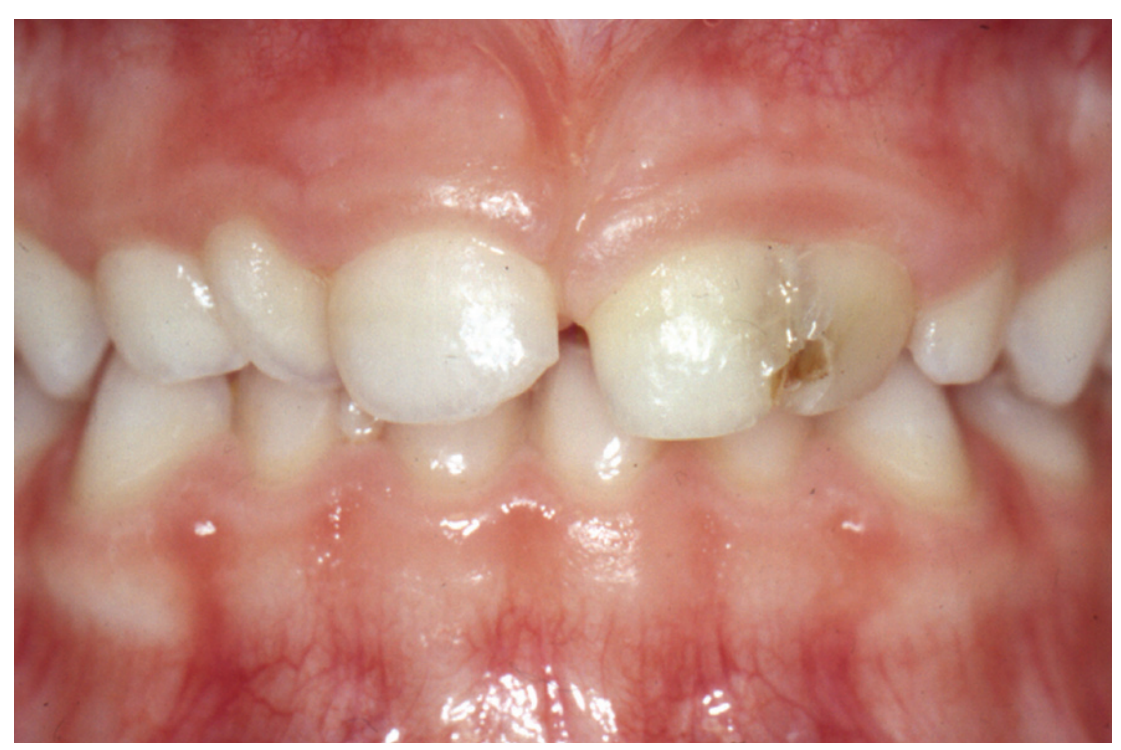

Figura 1. Caso 1. Fusión 6.1-6.2.

abre en abanico hacia incisal ${ }^{4}$. El tamaño del diente depende de la etapa en la que se produjo la fusión; los dientes fusionados tardíamente en el desarrollo a menudo producen dientes de tamaño doble del normal'.

La etiología y patogénesis de estas anomalías no están claras ${ }^{11-13}$, habiéndose involucrado factores traumáticos e inflamatorios que hayan afectado a ambos folículos". La etiología exacta no puede ser determinada por la dificultad para establecer las circunstancias embriológicas, ya que estas alteraciones resultan de acontecimientos anormales en el desarrollo embrionario del diente ${ }^{14}$. Algunos autores apuntan que la asociación de ciertas anomalías dentarias y algunos trastornos mentales sugiere la participación de la cresta neural en el desarrollo dental ${ }^{15}$. En otras investigaciones se encontró que altas dosis de vitamina A pueden causar anencefalia y fusión dental en ratas de laboratorio embarazadas $^{16}$. Hitchin y Morris des- cribieron la ontogenia de incisivos conoides y mostraron que el desorden primario es la persistencia de la lámina interdentaria. También mostraron casos de fusión dentro de miembros de una misma familia, lo que se podría atribuir a un probable patrón hereditario autosómico dominante, con un bajo grado de penetrancia ${ }^{11}$. Existe cierto grado de acuerdo en la literatura acerca de un posible componente hereditario para los dientes dobles en humanos $s^{4,5,11,17,18}$.

Los dientes fusionados pueden ser el resultado de alguna interacción física que hace que los gérmenes dentarios estén en íntimo contacto, con necrosis del tejido interdental ${ }^{9}$. Entre los factores etiológicos parece relevante la existencia de antecedentes traumáticos que podrían influir en la capacidad de unión de los dos gérmenes dentales durante las etapas pre-eruptivas.

Se han asociado otras posibles anomalías dentarias y no dentarias con este tipo de defectos, entre ellos ${ }^{18,19}$ 


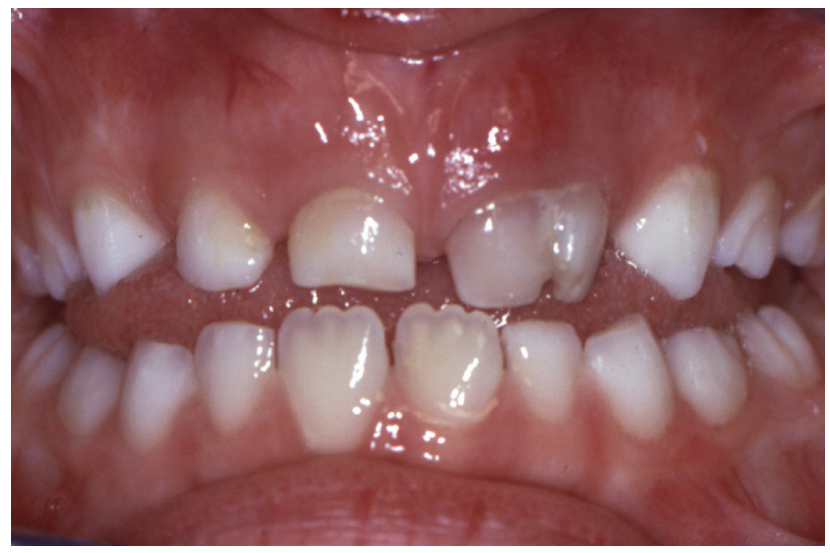

Figura 2. Caso 2. Fusión 6.1-6.2.

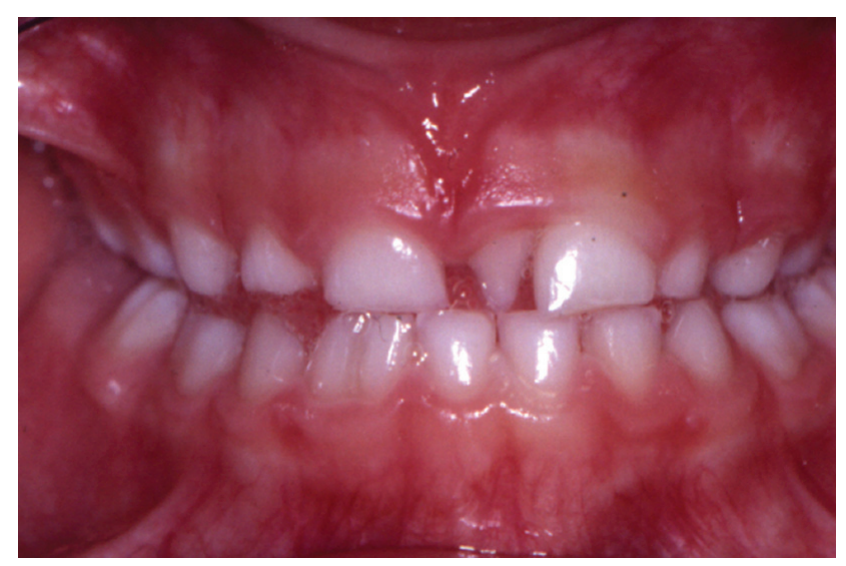

Figura 3. Caso 3. Fusión 8.2-supernumerario.
Ios dientes supernumerarios, hipodoncia, incisivos laterales superiores permanentes conoides, dens in dente, alteraciones en las uñas y sindactilia.

Entre las consecuencias clínicas cabe destacar que, al ocurrir generalmente en la región interincisiva, pueden provocar problemas estéticos de muy difícil solución si la fusión se extiende radicularmente ${ }^{1,2}$. Otros problemas que pueden estar asociados son la posible pérdida de longitud de la arcada y la erupción retrasada o ectópica de los dientes permanentes, así como la caries a lo largo de la línea de unión, y los abscesos periodontales $^{20-22}$.

\section{Casos clínicos}

Se describen a continuación siete casos clínicos de fusiones dentarias (tabla 1):

1. Paciente varón de 6 años que acude a revisión presentando a la exploración una fusión dentaria de 6.1 con 6.2. Presenta lesión de caries en el surco de unión de ambos dientes, así como en mesial de 6.2 y distal de 6.1 (fig. 1).

\begin{tabular}{|c|c|c|c|c|}
\hline Casosexo & Edad & Dentición & Hrmiarcada & Fusión \\
\hline Varón & 6 años & Temporal & Izquierda & Fusión $6.1-6.2$ \\
\hline Varón & 6 años & Temporal & Izquierda & Fusión $6.1-6.2$ \\
\hline Varón & 4 años & Temporal & Derecha & Fusión 8.2 - Supernumerario \\
\hline Mujer & 5 años & Temporal & Derecha & Fusión 8.1 - 8.2 \\
\hline Varón & 7 años & Temporal & Izquierda & Fusión $7.1-7.2$ \\
\hline Varón & 6 años & Temporal & Derecha & Fusión 8.2 - Supernumerario \\
\hline Mujer & 5 años & Temporal & Izquierda & Fusión $6.1-6.2$ \\
\hline
\end{tabular}

2. Paciente varón de 6 años de edad que presenta fusión de 6.1 con 6.2. Acude por absceso en la zona de vestíbulo bucal antero-superior izquierdo, presentando un absceso eruptivo de 2.1 que intenta reabsorber las raíces de ambos dientes fusionados (fig. 2).

3. Paciente varón de 4 años que acude presentando anomalías de unión y de número. A la exploración se detecta la presencia de un diente supernumerario mesiodens, así como la fusión del 8.2 con otro diente supernumerario (fig. 3).

4. Paciente mujer de 5 años que acude a revisión, detectando en la exploración una fusión de 8.1 con 8.2 (fig. 4).
5. Paciente varón de 7 años que acude con fusión de 7.1 con 7.2 (fig 5).

6. Paciente varón de 6 años que acude con fusión de 8.2 con un diente supernumerario (fig 6 ).

7. Paciente mujer de 5 años que acude a revisión y a la que se le detecta accidentalmente la fusión dentaria de 6.1 y 6.2 (fig. 7).

\section{Discusión}

\section{Diagnóstico diferencial}

Algunos autores catalogan las fusiones dentro de las anomalías de forma; sin embargo la alteración en el volumen y la forma del diente es la 


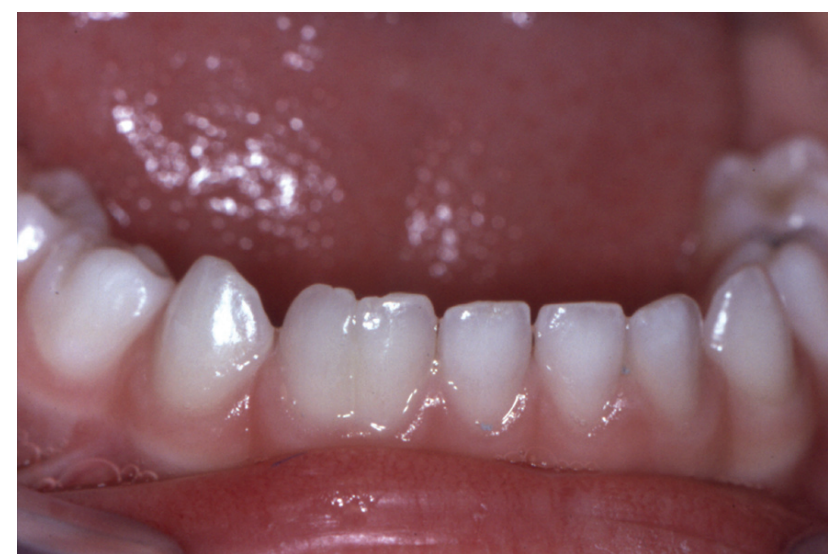

Figura 4. Caso 4. Fusión 8.1-8.2.

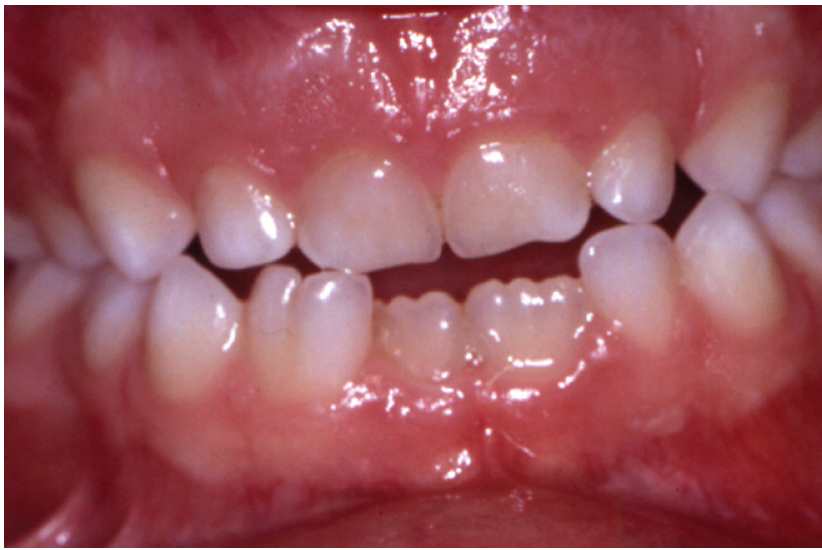

Figura 6. Caso 6. Fusión 8.2-supernumerario.

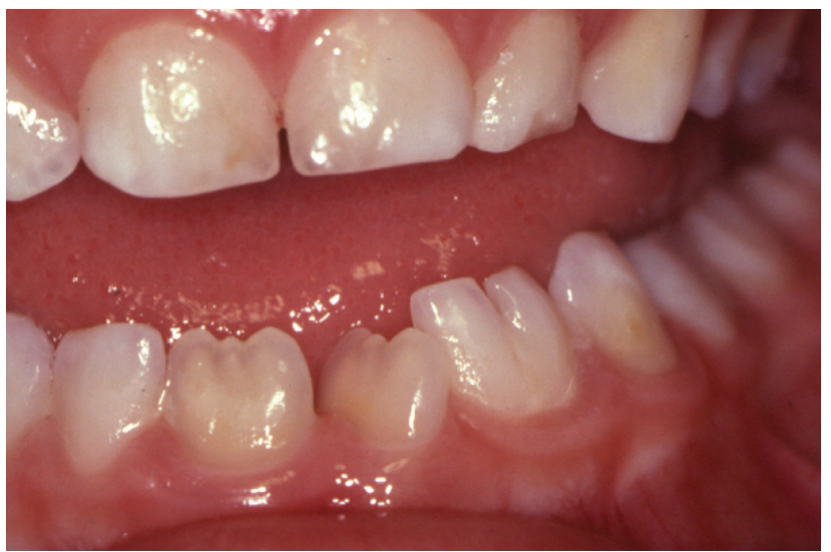

Figura 5. Caso 5 Fusión 7.1-7.2

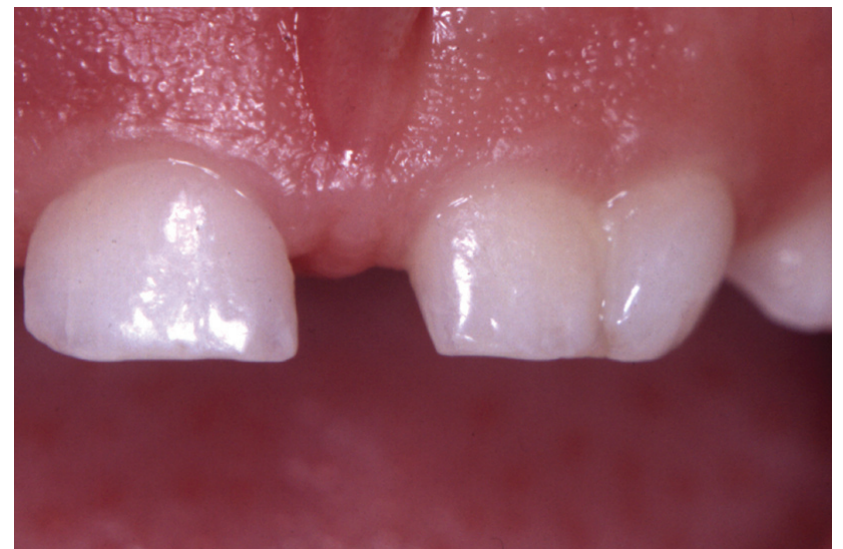

Figura 7. Caso 7. Fusión 6.1-6.2. consecuencia, pero no la causa de la anomalía, por lo que la fusión debe considerarse como anomalía por unión ${ }^{4}$. Algunas anomalías dentarias están definidas con precisión, pero este no es siempre el caso ${ }^{17}$, y dentro de las anomalías de unión ha habido cierta confusión entre diferentes entidades clínicas, sobre todo a la hora de diferenciar entre la fusión y la geminación $n^{7.23^{* *}}$, habiéndose utilizado también otros términos tales como diente doble (double teeth) o diente gemelo (dental twinning) para describir estas anomalías ${ }^{11,15,19,22,23^{* *}-25^{*}}$. Antes se utilizaba el término geminación como término universal para describir la fusión y la geminación, pero actualmente la fusión se reconoce comúnmente como la unión de dos gérmenes dentales en algún estadío de su desarrollo en el que se produce contacto de su dentina. Las cámaras pulpares y los conductos radiculares pueden estar unidos o separados, dependiendo de lo avanzado del desarrollo en el momento de la unión.

La geminación se define como un único germen dentario que intenta dividirse, resultando en un diente con una única raíz y una gran corona bífida, mientras que el término de fusión se refiere a dos o más gérmenes dentarios que forman un único diente con dos cámaras pulpares y sus respectivos conductos radiculares ${ }^{4}$.

El diagnóstico diferencial entre fusión y geminación es difícil, y posiblemente académico ${ }^{11,12,15,17,19,22,23^{* *}, 25^{*}, 26}$. Algunos autores sugieren contar los dientes como una ayuda en el diagnóstico: en la fusión suele haber un diente menos de lo normal al contar los de la arcada, si el diente afectado se cuenta como uno solo ${ }^{20,27-29}$. Si se la 
da un valor doble al diente fusionado, se obtendría un total correcto. Por el contrario, y según este método, al contar los dientes en un caso de geminación el resultado sería un número normal de dientes. Este método puede hacer difícil el diagnóstico diferencial entre fusión y geminación cuando hay una fusión entre un diente normal y un diente supernumerario, siendo la apariencia clínica en este caso similar a la de la geminación $n^{6,10}$ Sin embargo, raramente una fusión se presenta como una gran corona bífida con una cámara, situación que dificultaría el diagnóstico diferencial respecto a la geminación'. Kelly ${ }^{29}$ sugirió que en la geminación las dos mitades de las coronas unidas son usualmente imágenes especulares, en contraste con la fusión, que se manifiesta con una diferencia notable entre las dos mitades de la corona. Dependiendo de la fase en que se unan los gérmenes dentarios, la fusión puede ocurrir durante el desarrollo del esmalte, la dentina o el cemento. En el último caso se utiliza el término concrescencia ${ }^{17}$. También se debe considerar la macrodoncia en el diagnóstico diferencial en los casos de geminación o fusión completa, si bien las diferencias anatómicas entre los tres supuestos, unidas a los hallazgos radiológicos suelen facilitar el diagnóstico diferencial ${ }^{10}$. Esto corrobora que los únicos elementos diagnósticos que posee el clínico para poder realizar el diagnóstico final en estas anomalías son la exploración clínica y radiográfica ${ }^{4,7}$.
Normalmente las anomalías dentarias de unión, forma y número ocurren más frecuentemente en la dentición permanente, excepto la fusión, que afecta más comúnmente a la dentición primaria ${ }^{5}$. No se debe olvidar la problemática potencial debida a la tendencia de esta anomalía a repetirse en la dentición permanente $\mathrm{y} / \mathrm{o}$ a la ausencia congénita de los dientes sucesores permanentes, ya que no se ha establecido hasta ahora si estas secuelas están o no relacionadas con la fusión y geminación observadas en la dentición primaria.

\section{Formas clínicas y plan de tratamiento}

se han asociado varios problemas clínicos con estas anomalías dentarias, particularmente si se involucran los dientes anteriores, como es en la mayoría de los casos. Para el odontólogo restaurador existen una serie de problemas que pueden ser significativos y no fácilmente superables, como la apariencia estética, el apiñamiento, la acumulación de placa bacteriana secundaria a una superficie porosa irregular, siendo también frecuente la caries en el surco que divide una corona bífida ${ }^{10,12,14,30}$. Para el ortodoncista existen una serie de problemas adicionales que requieren cuidadosa consideración, tales como la interdigitación dental y reducción del resalte ${ }^{14}$. En pacientes con estas anomalías es muy importante el cuidado dental por motivos ortodóncicos, periodontales o estéticos.
A pesar de que en pocos casos de los descritos en la literatura se discute el plan de tratamiento, éste deberá ser tenido en cuenta especialmente cuando las anomalías dentarias se presenten en la dentición permanente ${ }^{10}$. Los incisivos de mayor tamaño influyen en el alineamiento anterior y en la simetría de la arcada, posiblemente causando serios problemas periodontales, ortodóncicos y estéti$\cos ^{19,24,30}$

Para prevenir la aparición de caries, el surco debe ser restaurado, y el surco palatino sellado ${ }^{10}$. En la dentición permanente se debe esperar a la completa formación radicular, pudiéndose posteriormente separar ambos dientes mediante desgastes selectivos de las superficies proximales y posteriormente restaurar mediante odontología adhesiva con resinas compuestas, dependiendo de la longitud de arcada y el espacio en la zona anterior maxilar. Esta separación se debe realizar tras la completa formación radicular debido a la posible comunicación entre las cámaras pulpares, dado que complicaría el tratamiento pulpar ${ }^{10}$.

En otros casos puede ser suficiente realizar restauraciones adhesivas de resinas compuestas para mejorar la estética. Si es necesario pueden hacerse desgastes selectivos en las zonas palatinas para evitar las interferencias oclusales ${ }^{10}$. Se deben aclarar con precisión las expectativas del paciente y la historia dental a la hora de determinar el planteamiento terapéutico más apropiado. 


\section{Bibliografia recomendada}

Para profundizar en la lectura de este tema, el/los autor/es considera/an interesantes los artículos que aparecen señalados del siguiente modo: *de interés * ${ }^{*}$ de especial interés.

1. Arrieta JJ, Bartolomé B. Anomalías dentarias. En: Varela M (editor). Problemas bucodentales en pediatría. Madrid: Ergón, 1999:43-58.

2. Villa MA. Patología pre-eruptiva. En: Bascones A (coordinador). Tratado de Odontología. Madrid: Trigo Ed, 1998;III:2459-63.

3. De la Macorra. Diagnóstico radiológico de las enfermedades dentarias. En: Bascones A (coordinador). Tratado de Odontología. Madrid: Trigo Ed, 1998;III:2931-38.

4. Nadal-Valldaura A. Patología dentaria. Barcelona: Ed Rondas, 1987.

5. Hagman FT. Anomalies of form and number, fused primary teeth, a correlation of the dentitions. J Dent Child 1988;55:359-61.

6. Chen R, Wang C. Gemination of a maxillary premolar. Oral Surg Oral Med Oral Pathol 1990;69:656.

7. Duncan WK, Helpin ML. Bilateral fusion and gemination: A literature analysis and case report. Oral Surg Oral Med Oral Pathol 1987;64:82-7.

8. Ravn JJ. Aplasia, supernumerary teeth and fused teeth in the primary dentition. Scand J Dent Res 1971;79:1-6

9. Munro D. Gemination in the deciduous teeth. Br Dent J 1958;104:238-40.

10. Mattos-Graner RO, Rontani RM, Gaviao MB, De Souza Filho FJ, Granatto AP, De Almeida OP. Anomalies of tooth form and number in the permanent dentition: report of two cases. ASDC J Dent Child 1997:64:298-302.

11. Hitchin AD, Morris I. Geminated odontome-con- nation of the incisors in the dog - its etiology and odontogeny. J Dent Res 1966;45:575-83.

12. Surmont PA, Martins LC, De Craene LG. A complete fusion in the primary human dentition: A histological approach. J Dent Child 1984;51:362-7.

13. O'Reilly PMR. A structural and ultrastructural study of a fused tooth. $\mathrm{J}$ Endodont 1989;15:442-6.

14. Chaudry SI, Sprawson NJ, Howe L, Nairn RI Dental twinning. Br Dent J 1997;182:185-8.

15. Puy L, Pizzarro C, Navarro F. Double teeth: Case reports. J Clin Pediatric Dent 1991; 15:120-4.

16. Knudsen PA. Fusion of upper incisors at bud or cap stage in mouse embryos with exencephaly induced by hipervitaminoses $\mathbf{A}$. Acta Odontol Scand 1965;23:549-65.

17. Levitas TC. Gemination, fusion, twinning, and concrescence. J Dent Child 1965;32:93-100.

18. Camm JH, Wood AJ. Gemination, fusion and supernumerary tooth in the primary dentition: Report of case. J Dent Child 1989;56:60-1

19. Killian CM, Croll TP. Primary and permanent incisor twinning defects in one dental quadrant: report of case. Quintessence Int 1990;21:363-5.

20. Milazzo A, Alexander SA. Fusion, gemination, oligodontia, and taurodontism. J Pedod 1982; 6:194-9.

21. Gersh RP, Isler S. Bilateral connation of primary molars: Report of a case. J Dent Child 1973;40:39-41.
22. Mader CL. Fusion of teeth. J Am Dent Assoc 1979;98:62-4.

$23^{* *}$. Schurs A, van Loveren C. Double teeth: review of the literature. J Dent Child 2002, 67:313-25.

Completa y actualizada puesta al día sobre las anomalías dentarias de dientes dobles, en la que se establece claramente el diagnóstico diferencial de las mismas.

24. Reisenberg RE, Killian CM. Triplication and twinning in one dental arch: report a case. Quintessence Int 1990;21:621-3.

$25^{*}$. Killian CM, Croll TP. Dental twinning anomalies: the nomenclature enigma. Quintessence Int 1990;21:571-6.

Revisión sobre las diferentes formas clínicas de dientes dobles y su diagnóstico diferencial.

26. Trubman A, Silberman SL. Triple teeth: case reports of combined fusion and gemination. J Dent Child 1988;55:298-9.

27. Gellin ME. The distribution of anomalies of primary anterior teeth and their effect on the permanent successors. Dent Clin North Am 1984;28:69-80.

28. Croll TP, Rains NJ, Chen E. Fusion and gemination in one dental arch: Report of a case. $J$ Dent Child 1981;48:297-9.

29. Kelly JR. Gemination, fusion or both? Oral Surg Oral Med Oral Pathol 1978;45:655-6.

30. O'Reilly PMR. Structural and radiographic evaluation of four cases of tooth fusion. Aust Dent J 1990;35:223-6. 